\title{
Erratum: First-principles calculation of nonlinear optical responses by Wannier interpolation [Phys. Rev. B 96, 115147 (2017)]
}

\author{
Chong Wang ${ }^{\circledR}$, Xiaoyu Liu, Lei Kang, Bing-Lin Gu, Yong Xu, and Wenhui Duan
}

(Received 10 May 2020; published 8 June 2020)

DOI: 10.1103/PhysRevB.101.249901

Four minor errors and typographical errors in the original paper are corrected as follows.

(I) Equation (5) should be corrected as

$$
r_{n m ; a}^{b}=\frac{i \hbar^{2}}{E_{n m}}\left[\frac{v_{n m}^{b} \Delta_{n m}^{a}+v_{n m}^{a} \Delta_{n m}^{b}}{E_{n m}}+\sum_{p \neq n, m}\left(\frac{v_{n p}^{b} v_{p m}^{a}}{E_{p m}}-\frac{v_{n p}^{a} v_{p m}^{b}}{E_{n p}}\right)\right]+\frac{1}{i E_{n m}}\left\langle n\left|\partial_{a} \partial_{b} \hat{H}(\mathbf{k})\right| m\right\rangle(n \neq m) .
$$

This equation was not used in the calculations.

(II) Throughout the original paper, $e$ is the charge of electron, such that $e=-|e|$. Unfortunately, the authors made a sign error in the code for the shift current. Therefore, the lines in Figs. 1(c) and 1(d) and Figs. 3(b)-3(d) should be flipped with respect to the horizontal axis. The results of Ref. [9] should also be flipped due to a different choice of the coordinate system. Therefore, our result and Ref. [9]'s result are still consistent with reasonable accuracy. For the reader's convenience, we plot the corrected figures in Figs. 1 and 2 here.

(III) The interband term of second harmonic generation in Appendix A should be

$$
\chi_{e}(\omega)=\frac{e^{3}}{2 \hbar^{2}} \sum_{n m l} \int \frac{d^{3} k}{(2 \pi)^{3}} \frac{r_{n m}^{a}\left(r_{m l}^{b} r_{l n}^{c}+r_{m l}^{c} r_{l n}^{b}\right)}{\omega_{l n}-\omega_{m l}}\left[\frac{2 f_{n m}}{\omega_{m n}-2 \omega}+\frac{f_{l n}}{\omega_{l n}-\omega}+\frac{f_{m l}}{\omega_{m l}-\omega}\right] .
$$

This typographical error did not enter the code and the relevant calculations were correct.

(a)

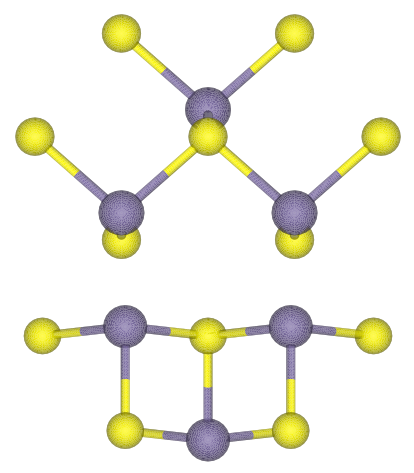

(c)
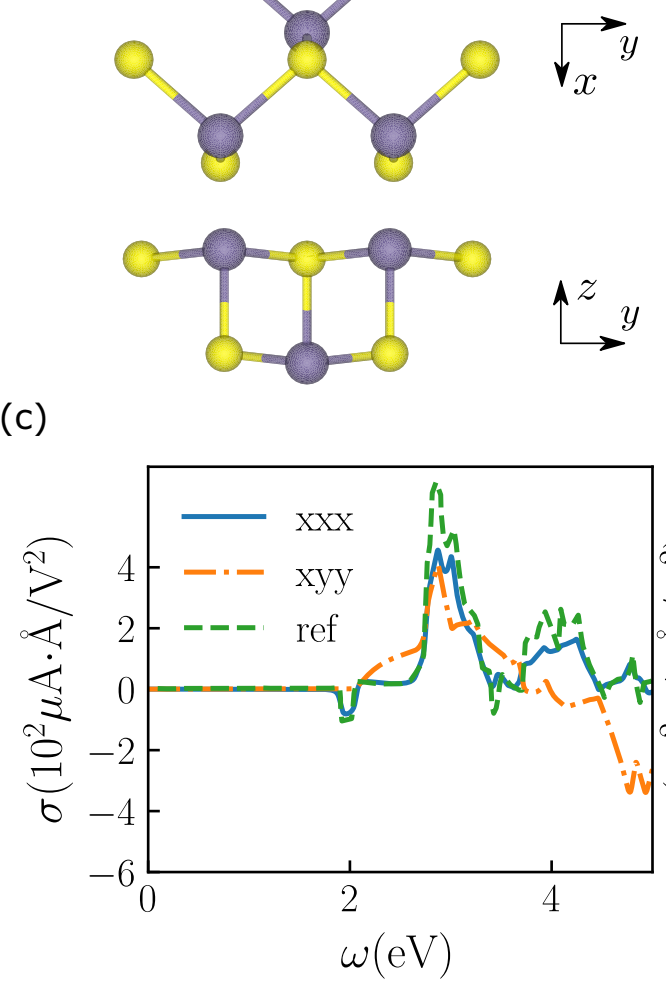

(b)

(d)
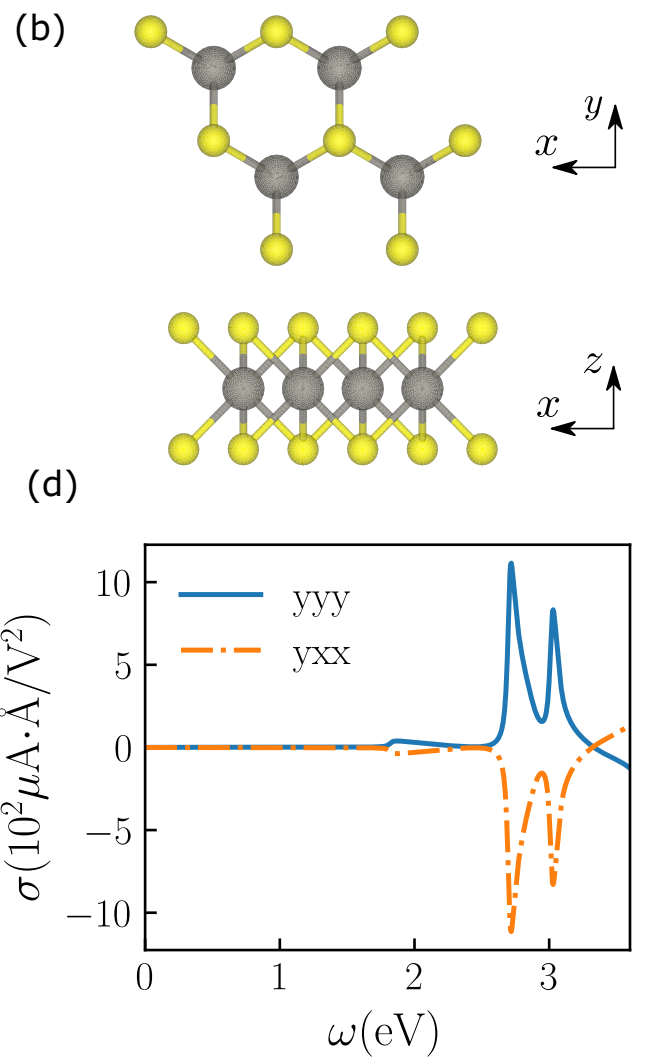

FIG. 1. The corrected Fig. 1 of the original paper. 


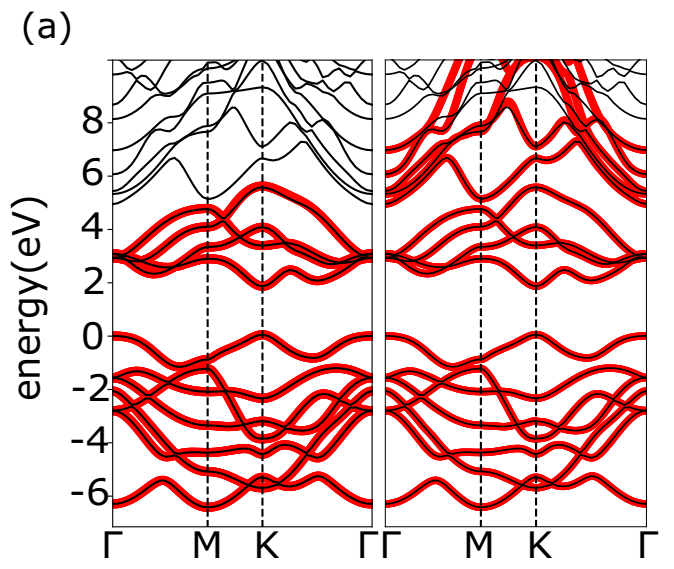

(c)

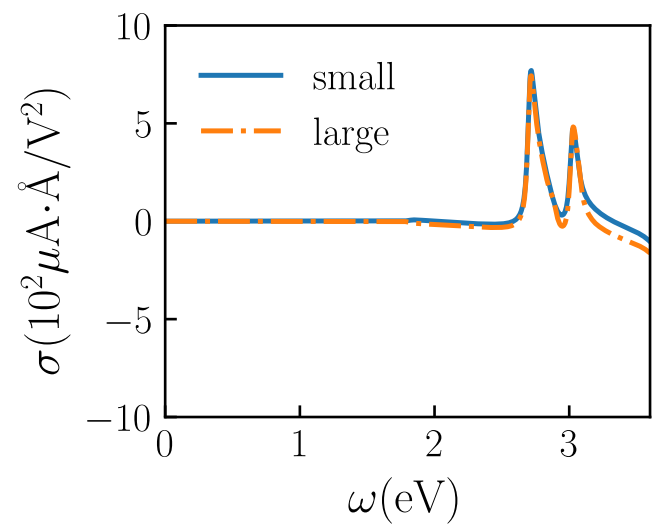

(b)

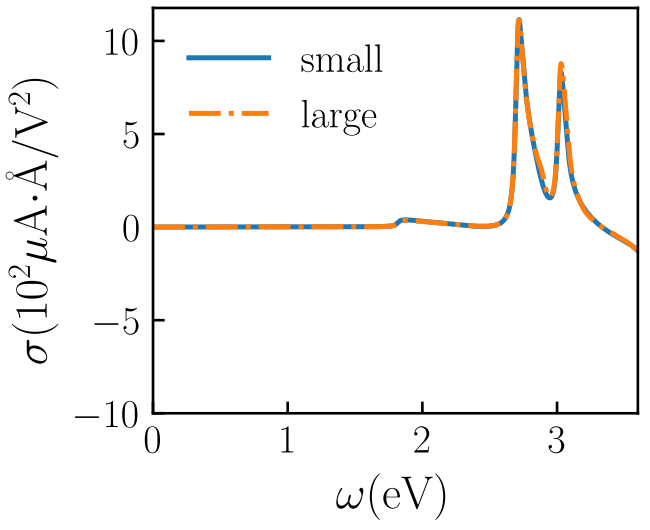

(d)

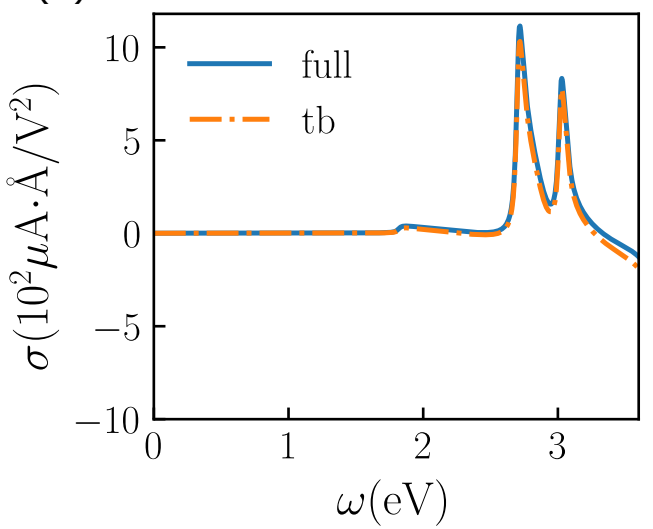

FIG. 2. The corrected Fig. 3 of the original paper.

(IV) For $n=m$, Eq. (8) should be clarified with

$$
\left(U^{\dagger} \partial_{a} \partial_{a} U\right)_{n m}=-\sum_{l \neq m} \frac{\left\langle m\left|H_{a}^{(\mathrm{W})}\right| l\right\rangle\left\langle l\left|H_{a}^{(\mathrm{W})}\right| m\right\rangle}{\left(E_{l}-E_{m}\right)^{2}} .
$$

This equation in the original paper was correct. However, the subscript $n$ on the left-hand side of the equation was reused as a summation index on the right-hand side, which may cause confusion for the readers. Similarly, for $a \neq b$ and $n=m$, Eq. (8) should be presented as

$$
\left(U^{\dagger} \partial_{b} \partial_{a} U\right)_{n m}=-\frac{1}{2}\left(\sum_{l \neq m} \frac{\left\langle m\left|H_{b}^{(\mathrm{W})}\right| l\right\rangle\left\langle l\left|H_{a}^{(\mathrm{W})}\right| m\right\rangle}{\left(E_{l}-E_{m}\right)^{2}}+\sum_{l \neq m} \frac{\left\langle m\left|H_{a}^{(\mathrm{W})}\right| l\right\rangle\left\langle l\left|H_{b}^{(\mathrm{W})}\right| m\right\rangle}{\left(E_{l}-E_{m}\right)^{2}}\right) .
$$

The authors are grateful to the following people: Andrew Smith pointed out the typo in (I); Meng Ye and Nianlong Zou confirmed the sign problem in (II); and Yang Zhang pointed out the typographical error in (III). 